\title{
Pleasures of Ambiguity: the Case of Piranesi's Carceri
}

\author{
Johan Wagemans ${ }^{1, *}$, Jan Koenderink ${ }^{1,2}$ and Andrea van Doorn ${ }^{3}$ \\ ${ }^{1}$ University of Leuven (KU Leuven), Laboratory of Experimental Psychology, \\ Tiensestraat 102, P.O. Box 3711, BE-3000 Leuven, Belgium \\ ${ }^{2}$ Utrecht University, Experimental Psychology, Willem C. van Unnik Building, \\ Heidelberglaan 2, 3584 CS Utrecht, The Netherlands \\ ${ }^{3}$ Delft University of Technology, Industrieel Ontwerpen, Postbus 5, \\ 2600 AA Delft, The Netherlands
}

Received 5 January 2013; accepted 19 February 2013

\begin{abstract}
Are pictorial spaces evoked by intentionally ambiguous pictures internally coherent? If so, then we expect these to be idiosyncratic. We addressed this issue empirically. Three sheets from the second edition of Piranesi's Carceri d'Inventione were selected for the experiment. These were compared with a conventional landscape drawing (after a Capriccio by Guardi) as a base line. The pictorial spaces were probed by way of pairwise depth-order judgments. In each image about fifty landmarks were selected, thus yielding over a thousand binary depth-order judgments per image. From such a full set of pairwise comparisons one obtains a linear depth order. This linear order again yields postdictions for the individual pairwise judgments. Actual judgments differ from these postdictions, thereby yielding a convenient handle on the coherence of the pictorial space. Since the Piranesi Carceri are generally considered to be 'difficult', 'ambiguous' or 'labyrinthine', whereas a conventional landscape as the Guardi is designed for its well-defined depth structure, it is expected that they will give rise to very distinct degrees of coherence. This is indeed what we find. We studied the variation of coherence over observers and images in detail. The pictorial locations that are heavily involved in incoherent responses were identified, and the reason for their ambiguous nature traced in the structure of the depth cues provided by the artist (Piranesi). We speculate that the Carceri manage to strike just the right level of ambiguity (between total chaos and obvious structure) so as to render them visually attractive.
\end{abstract}

\section{Keywords}

Perceptual ambiguity, pictorial space, depth perception, perceptual coherence, visual interest, visual attraction, order and complexity

\footnotetext{
* To whom correspondence should be addressed. E-mail: johan.wagemans@psy.kuleuven.be 


\section{Introduction}

Pictorial space is an entity of considerable conceptual interest (Koenderink and van Doorn, 1995, 2003; Koenderink et al., 2011). It is the three- (or, perhaps, two-plus-one-) dimensional space that dominates your visual awareness when you look into a picture, as opposed to looking at that picture. When you look at a picture, you are aware of a flattish object in the space you move in, that happens to be covered with a pattern of pigments. When you look into a picture, you are aware of a space that is 'elsewhere' or 'in the mind' (Pirenne, 1970). Neither your eye, nor the picture surface is located in this 'pictorial space'. Usually you are aware of both the space you move in and the pictorial space. This dual awareness produces a tension that is one of the charms of pictorial art.

'Pictorial space' is also of interest because it results from 'monocular stereopsis' (Gombrich, 1961; Graham, 1965; von Helmholtz, 1856). The space in the picture is due to the 'depth cues' (used as a generic term for a broad spectrum of things) that you somehow 'pick up' or 'resonate with'. In a painting, they are often due to pictorial structures that were intentionally provided by the painter. In a holiday snapshot, they may just happen to be suggested to you by the structure of the photograph, but in an artistic rendering nothing is accidental, everything is intentional. In monocular vision of a real scene, the depth cues picked up by you may be similar to the ones indicated above. This suggests that 'monocular stereopsis' in real scenes is not unlike pictorial vision (Hildebrand, 1893). The major difference is that, in front of a real scene, you are not simultaneously aware of the presence of any 'picture plane'. Thus, the study of pictorial vision is not only of interest for its own sake, but also because it offers a handle on the study of monocular stereopsis per se.

We have developed an extensive toolbox of methods that allows us (like anyone ready to adopt our methods) to address the pictorial space experienced by human observers in quantitative detail (van Doorn et al., 2011; Wagemans et al., 2011a, 2011b). These methods have been shown to yield measurements (typically 'depth maps') that are internally consistent, and in mutual agreement. Here 'in agreement' should be understood as 'modulo some idiosyncratic mental movement', where the group of 'mental movements' is formally defined. If two depth maps are 'in agreement', this formalism implies that one can find a map that depends upon only three parameters, which will yield a coefficient of determination for the correlation of depths at corresponding locations of over 0.9 , and usually more like 0.99 . Notice that this is hardly trivial, because 'straight' depth correlations often yield values that are not significantly different from zero.

The experiments to which the above discussion refers were done on pictures (photographs, paintings, full drawings, or mere cartoon drawings) that 
were 'coherent' and typically considered 'realistic'. It stands to reason that one should expect less coherent results for pictures that are intrinsically ambiguous. Indeed, we have found this to be the case in a study involving mere silhouettes. Recently we studied reputedly 'difficult' drawings by Picasso involving multiple simultaneous viewpoints - and found that although observers agree quite well on parts, they fail to do so on the drawing as a whole (Koenderink et al., 2012).

In the present study we intentionally use pictures that are frankly globally inconsistent, because the artist intentionally introduced ambiguities and contradictions. The expectation is, of course, that the results will prove to be internally inconsistent too, and that the mutual agreement between observers will diminish very significantly.

\section{Methods}

\subsection{Stimuli}

The stimuli were sheets from Giovanni Battista Piranesi's (1720-1778) series of etchings known as the Carceri d'Inventione (Imaginary Prisons), or just Carceri (see Note 1) (Ficacci, 2000; Hofer, 1973). The Carceri is a series of sixteen sheets (published in first and second states) that depict labyrinthine vaults with numerous unexpected views, harboring immense machines that remind one of the inquisition torture chambers. The scale is especially striking. Piranesi started work on the series in 1745, and published the first set of prints (fourteen, unnumbered sheets, size $16^{\prime \prime} \times 21^{\prime \prime}$ ) in 1750 . The second printing was in 1761. Piranesi worked up all the plates, and added two new ones (II and V). The sheets were numbered I-XVI. In reworking the plates he evidently intentionally increased the ambiguity by adding inconsistent cues.

For the experiment we selected Plates V ('The lion bas-reliefs'), VI ('The smoking fire') and XI ('The arch with shell ornament'). Plates V and VI have portrait, plate XI landscape aspect ratio. We refer to these pictures as 'Stimulus 1' (Plate V), 'Stimulus 2' (Plate VI) and 'Stimulus 3' (Plate XI). Stimuli 2 and 3 are reworked versions (second edition) of the original series; Stimulus 1 is one of the plates added in the second edition.

As a comparison we used a wash drawing after an original Capriccio by Francesco Guardi (1712-1793) (see Note 2). The Capriccio shows an imaginary landscape based on the generic Vedute from the Veneto region. It was selected because it has a generic (foreground-middle ground-background) structure, and uses the familiar bouquet of depth cues. We expect all observers to come up with essentially the same depth map in this case. Thus the Guardi provides a baseline for the (in all likelihood much more incoherent) Piranesi data. 
The pictures are presented on a computer screen (DELL U2410f monitor, $1920 \times 1200$ pixels liquid crystal display (LCD) screen), each picture filling the screen as much as possible. In viewing the pictures the observers are positioned at a fixed distance $(78 \mathrm{~cm})$, centered with respect to the screen. They view the screen monocularly. Apart from the screen, the room was in darkness.

\subsection{Observers}

We have six observers, AD, EG, JK, JW, MS and RK, three of these (AD, JK, JW) being authors. The observers include both genders, and ages from midtwenties to late sixties. All observers were emmetropic or used their regular prescription spectacles. They used their dominant, or preferred, eye, the other eye being patched.

\subsection{Set Up and Methodology}

The method we used was perhaps the simplest possible, namely pairwise depth comparison. This method has been studied in detail before (van Doorn et al., 2011). We defined 49 points on each picture, yielding 1176 orderless pairs (Fig. 1). For each pair, the observer decides which location 'is closer'. A session takes about an hour and a half. Most observers find the task rather interesting initially, but more boring afterwards, when a large number of trials have been carried out on the same stimulus.

From this full data set we construct the linear order that best explains the data. From this linear order we again predict pairwise depth orders. These can be compared to the data. Thus, we find a linear order and a set of disagreements for each observer.

\section{Experiments}

The fiducial points (Fig. 1) were designated by the authors. We selected evident 'landmarks', and distributed these (in an informal fashion) roughly uniformly over the picture area and the depth ranges. Since we distributed almost fifty points, we feel confident that there is unlikely to be a selection bias: after distributing half of the points one starts to find it harder and harder to place the remainder. It is likely that another attempt would yield an appreciable overlap.

There were four major groups of experiments, namely:

Experiment 1: All observers completed a session on the Guardi stimulus. This experiment was designed as a baseline for Experiments 2 and 3.

Experiment 2: All observers completed a session on the Piranesi Stimulus 1 . These data can immediately be compared to the results of Experiment 1 . Moreover, they yield a handle on inter-observer variations. 

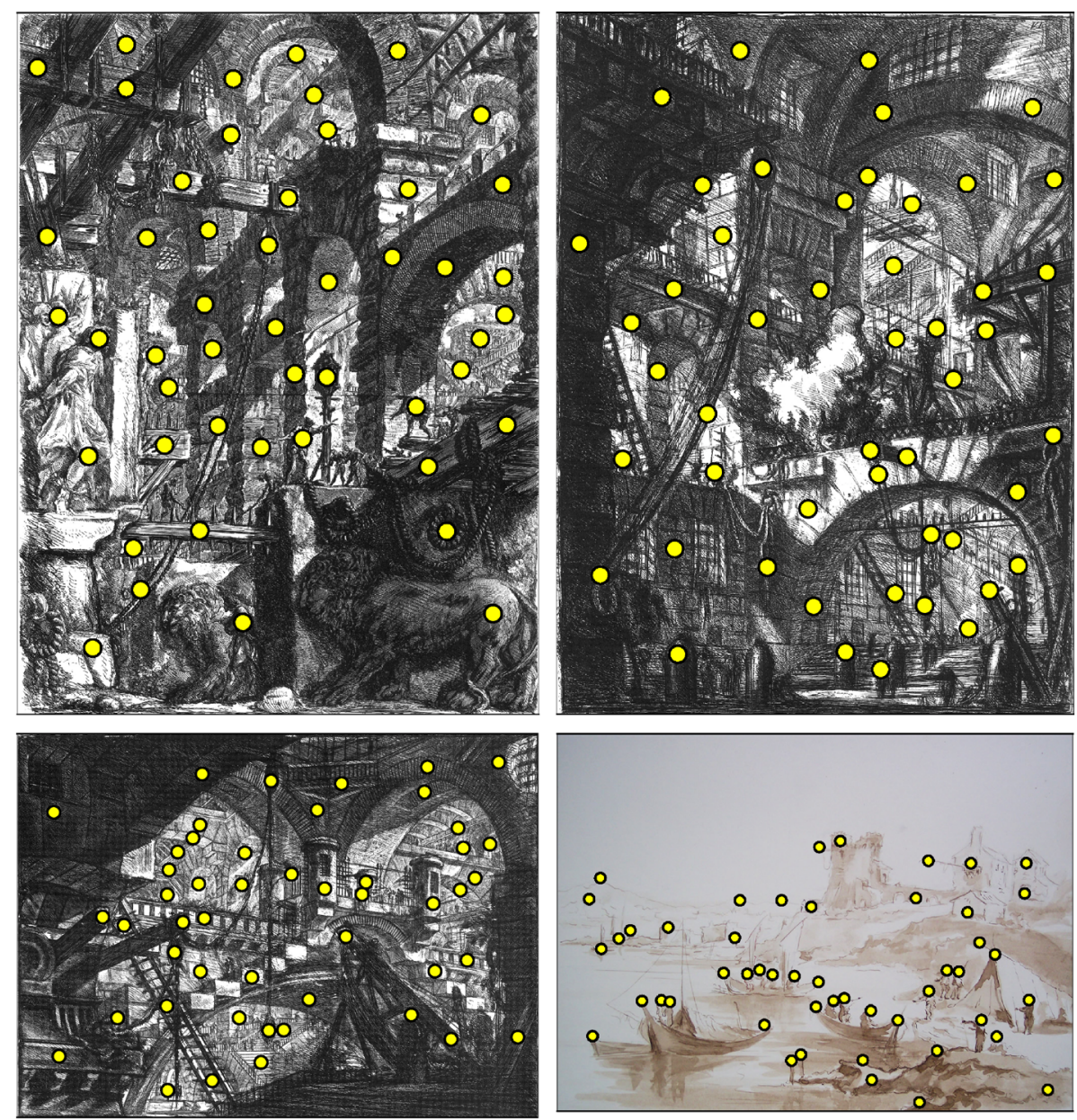

Figure 1. The fiducial points for the Piranesi Stimuli 1 (top left), 2 (top right), 3 (bottom left) and the Guardi stimulus (bottom right).

Experiment 3: Observers AD, JK and JW completed a session on all Piranesi stimuli. These data yield a handle on inter-observer variations, and stimulus related differences.

Experiment 4: Observer AD completed six sessions on all Piranesi stimuli. The sessions were done with considerable time interval (weeks), in order to improve the mutual independence. This experiment yields some insight into intra-observer variability.

These experiments allow many important comparisons to be directly made. The total data volume is quite large, amounting to over forty-thousand binary decisions. 


\subsection{Experiment 1}

In the case of the Guardi stimulus observers had little trouble with their decisions. For any fiducial point one obtains a clear-cut division into nearer and farther points (Fig. 2).

We have a full data matrix, since all possible point pairs have been judged once. The first analysis consists in finding a permutation of the points that renders the data matrix as diagonal as possible (Fig. 3). A simple algorithm suffices for this: a linear order is established by simply counting for each point how many points were judged to be in front of it. This algorithm can be shown to be equivalent to an optimal (in the least squares sense) depth-order estimate. Of course, occasional ties are to be expected.

From the linear order we predict (or, if you want, 'postdict') the individual judgments, and compare them to the actual judgments. We find a typical error rate of about one percent. The quality of the result can be expressed in terms of a 'number of merit' defined as the number of pairs minus the number of errors, divided by the number of pairs. For the example (session of observer $\mathrm{AD}$ ) we find a figure of merit of 0.98. It is typical for all observers (EG 0.90, JK 0.98, JW 0.96, MS 0.97, RK 0.97).

\subsection{Experiment 2}

In the case of the Piranesi stimuli the error rate is much higher (Fig. 4). In the case of Stimulus 1, Session 1 of observer AD, we have a median figure of merit of 0.84 , the error rate being more than eight times as large as for the

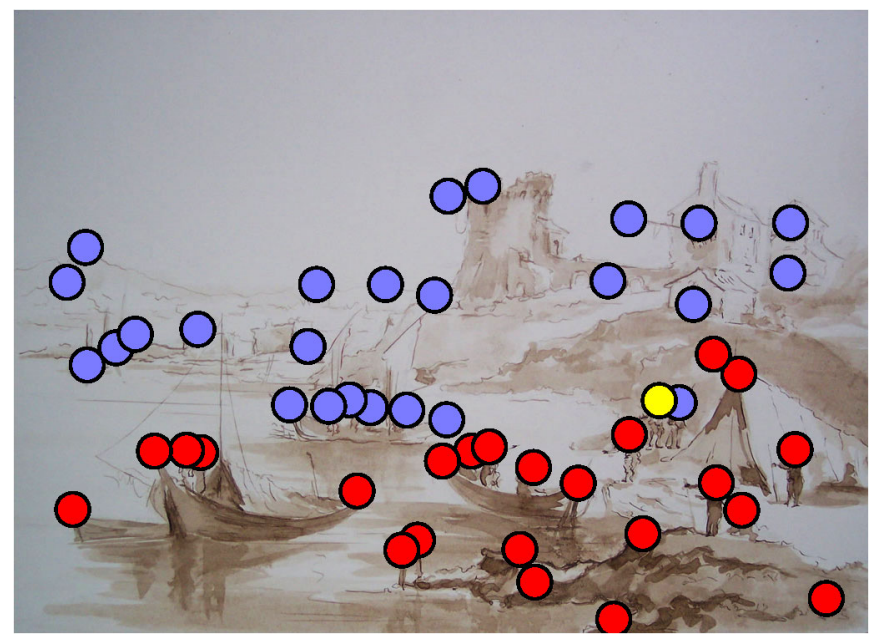

Figure 2. The points judged in front (red) or behind (blue) for the fiducial point shown in yellow for the Guardi stimulus. 


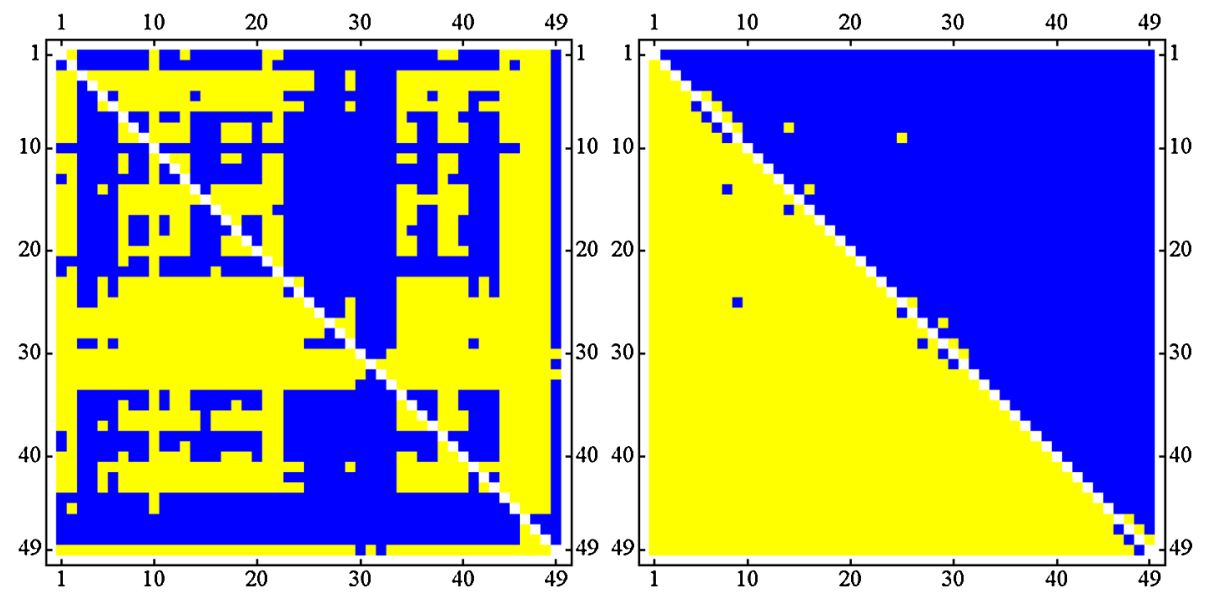

Figure 3. At left the data matrix for the Guardi stimulus, session of observer AD. At right the data matrix after the optimal point permutation, resulting in a linear depth order. Although the raw data matrix (at left) depends upon the - merely incidental - order of presentation, the sorted one (at right) reveals a non-incidental property of the observer.

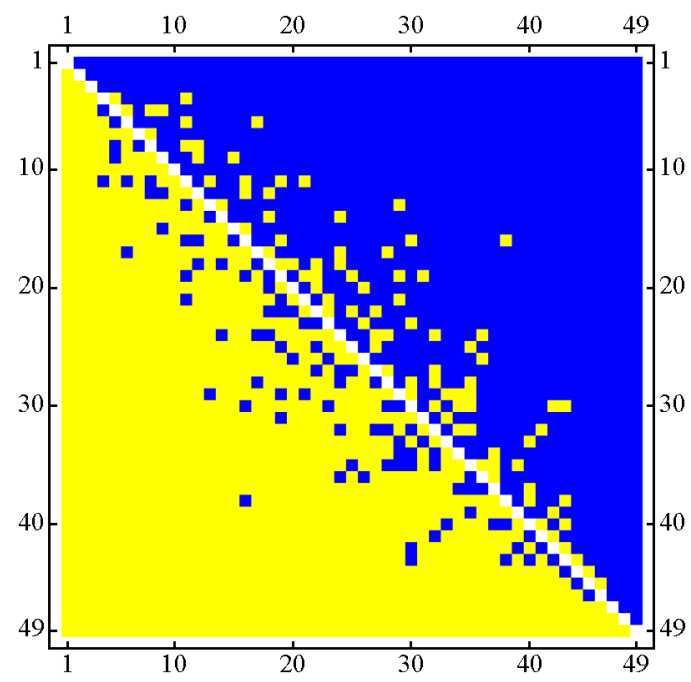

Figure 4. The sorted data matrix for the Piranesi Stimulus 1, Session 1 of observer AD. Notice the frequent inconsistencies as compared with the Guardi (Fig. 3, right).

Guardi stimulus. This is typical for all observers (EG 0.73, JK 0.85, JW 0.83, MS 0.85, RK 0.83).

\subsection{Experiment 3}

Three observers (AD, JK and JW) completed one session on all three Piranesi stimuli. For all Piranesi stimuli, and all observers, the figures of merit are low. 
The highest figure of merit for the Piranesi images is 0.895 (lowest 0.840 ), whereas the lowest figure of merit for the Guardi is 0.923 (highest 0.983). Thus, the distinction is categorical.

\subsection{Experiment 4}

In this case we have six sessions for each stimulus. Thus, it is possible to compute an overall best linear order over all sessions, and relate the individual judgments to that. We may relate error rate and figure of merit to this. We find that the quartiles for the figure of merit for observer $\mathrm{AD}$, over all six sessions are $0.857,0.860,0.872$ for Stimulus 1, 0.835, 0.844, 0.857 for Stimulus 2, and 0.864, 0.869, 0.888 for Stimulus 3.

\section{Analysis}

\subsection{Comparison of Experiments 1 and 2}

As might have been expected, there is a very significant difference between the data for the Piranesi Stimulus 1 as compared to that for the Guardi stimulus. The figures of merit for the Piranesi Stimulus 1 are lower than those for the Guardi stimulus (Fig. 5).

Another useful measure of the coherency of the results is the "determinacy index', which we define (for any given location) as the fraction of the 48 judgments that agree with the prediction according to the best linear order over all observers. We find that the distributions for the determinacy index for the two stimuli are very different: the interquartile ranges for the Guardi are 0.93-0.95; for the Piranesi 1 they are $0.67-0.81$.

These two measures are both needed. The 'figure of merit' is a measure of intrinsic consistency, whereas the 'determinacy index' is extrinsically determined with respect to some fiducial linear order. Thus the figure of merit is used to assess single sessions, whereas the determinacy index applies to pairs of sessions, or the relation of a single session to a collection of similar sessions (e.g., for various observers, or a single observer on repeated occasions).

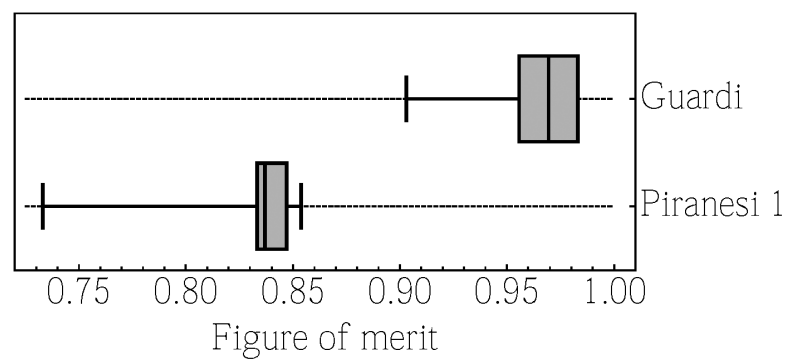

Figure 5. The figures of merit over all observers (for observer AD only the first session for the Piranesi Stimulus 1 is used). The whisker box plots show quartiles and extreme values. 
Thus, we may conclude that the Piranesi Stimulus 1 gives rise to much less coherent pictorial space than a conventional landscape rendering like the Guardi stimulus. It is perhaps satisfactory that this objective result corroborates centuries of intuitive insight.

\subsection{Experiment 2: Inter-observer Comparisons and 'Ambiguous Locations'}

For Piranesi Stimulus 1 we have one session for each observer (Fig. 6). After finding the best linear depth sequence over all data, we obtain the determinacy indices for the individual observers. These range from 0.653 to 0.784 (median 0.755, interquartile range 0.753-0.774). A relative outlier is perhaps observer EG (determinacy index 0.653). An analysis of observer EG's data alone yields a figure of merit of 0.733 , lower than that of the other observers

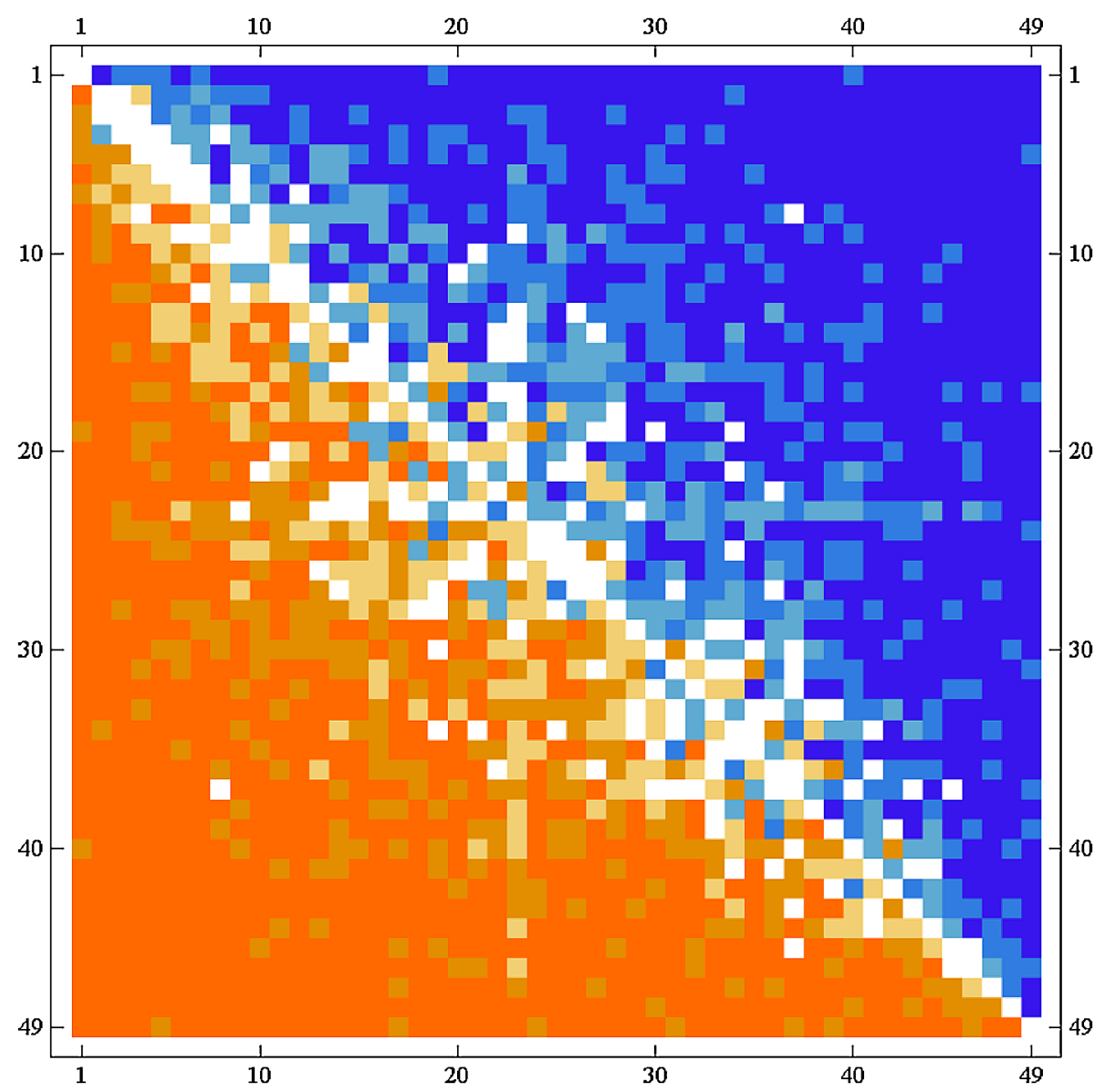

Figure 6. The data matrix (after the optimal diagonalization) for the sessions of six observers, Piranesi Stimulus 1. Notice that there are numerous unresolved inconsistencies. 
(range 0.833-0.854). Apparently, observer EG issues judgments that are systematically somewhat different from that of the majority opinion.

An analysis of non-explained judgments might conceivably yield an insight into the possible existence of 'ambiguous locations'. First we select the pairs for which four or more mismatches between the individual judgments and the prediction based on the overall best linear depth order are counted. There are 33 (out of 1176) of such pairs. Then we count how often a location is a member of such a pair. We find that the frequency ranges from zero to five. Then we select the locations that occur at least three times among the 'bad pairs'. We end up with ten locations. These locations are likely to be very 'ambiguous' with regard to their 'depth' (Fig. 7).

A very similar measure can be based on the 'determinacy index'. We find that the locations belonging to pairs with the lowest determinacy index tend to agree with the set of ambiguous points as defined above. (In both cases we take the initial ten points or so.)

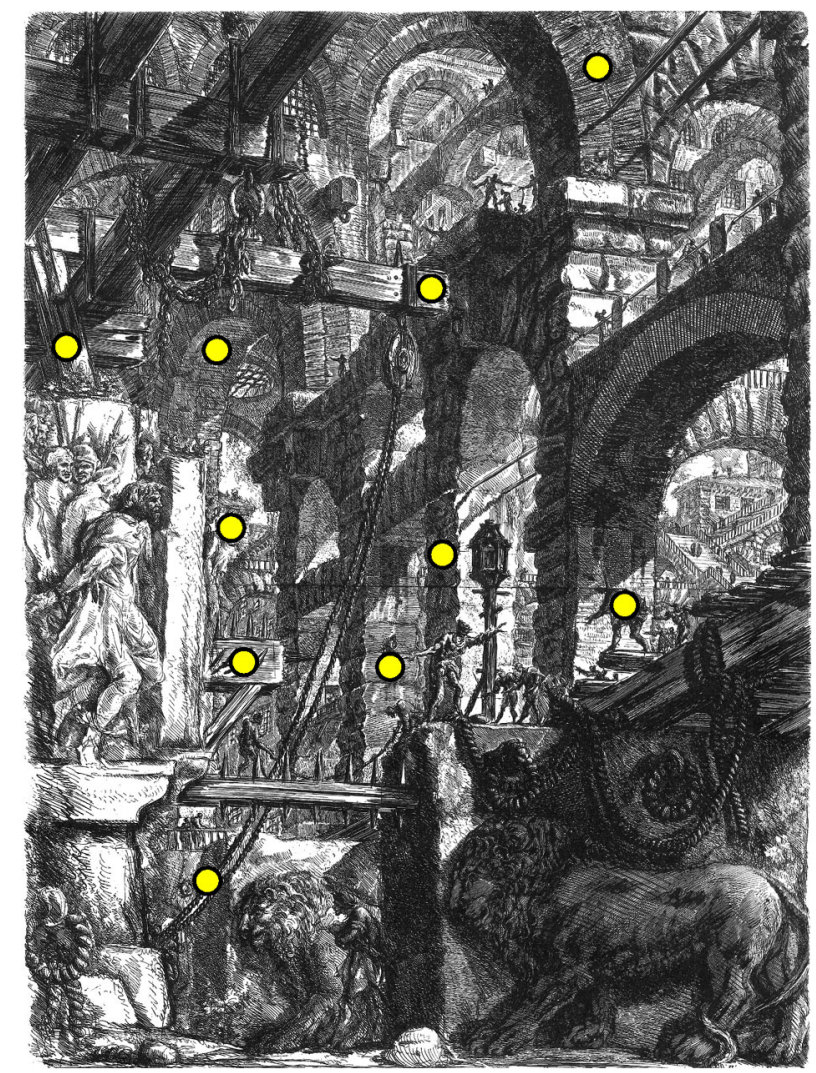

Figure 7. The 'ambiguous points' based on the sessions of six observers, Piranesi Stimulus 1. 


\subsection{Experiment 3: Mainly Stimulus Dependency}

For the three observers AD, JK and JW we may find the sets of 'most ambiguous locations' for all three stimuli. We aim for sets of about ten points, a fifth of the total number. This seems intuitively reasonable in view of the general performance of the observers. For the majority of points they mostly agree for pairs composed of 'good' points, but the 'ambiguous' points are involved in frequent mismatches, no matter whether they occur in combination, or whether the pair includes only one. Thus, it seems very likely that these ambiguous points may be useful in a search for the possible causes of the (generally agreed upon) labyrinthine character of Piranesi's Carceri prints.

Perhaps the most striking property of the ambiguous locations (Figs 7 and 8) is that they are not immediately connected to surfaces, but tend to stand relatively 'free' in space.

Just consider:

Piranesi 1: one point is located on the central hanging rope, one point is located on the end of the beam carrying the rope, one is a figure standing on a cantilevered bar;

Piranesi 2: two points are on hanging ropes, one on the hanging lamp (?), two others are on beams sticking out from machinery;
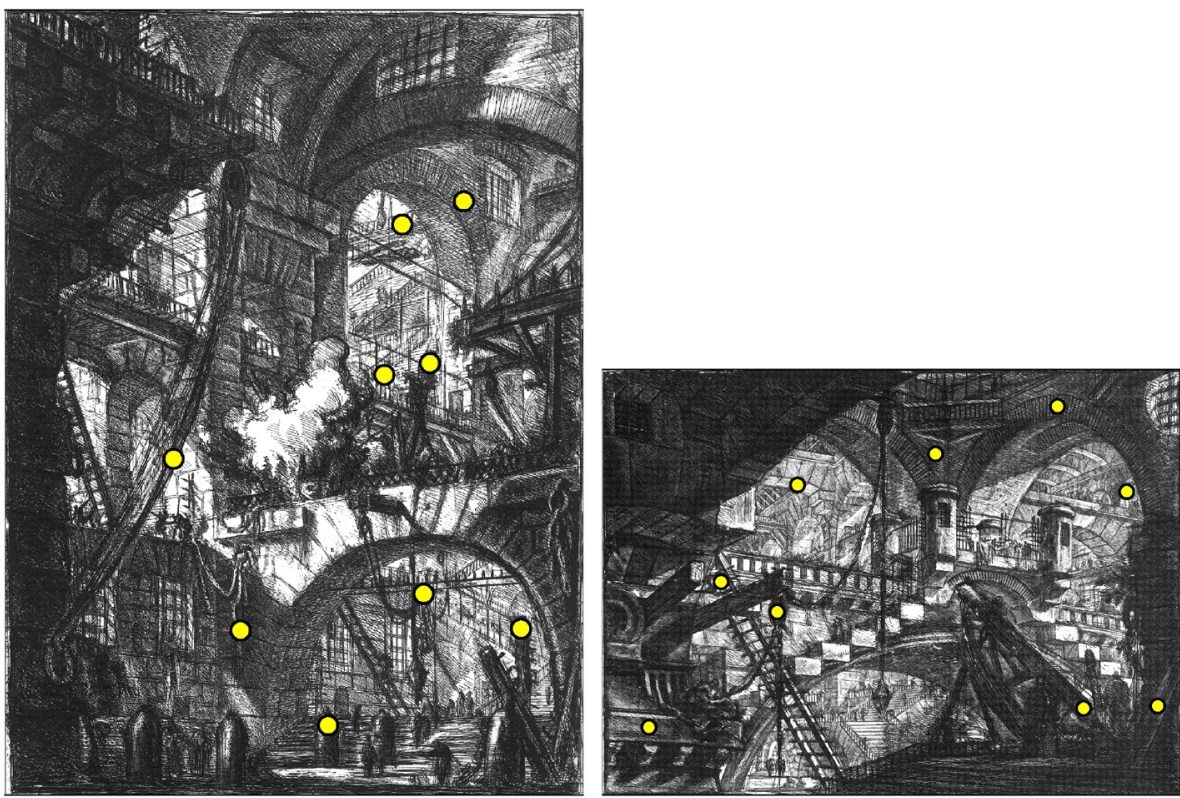

Figure 8. The most ambiguous points for Piranesi Stimulus 2 and Stimulus 3, based on sessions of the observers AD, JK and JW. (For the case of Piranesi Stimulus 1, the most ambiguous points for these observers agree with these obtained for the full set of six observers.) 
Piranesi 3: one point on a hanging pulley, one on an obliquely leaning ladder, two on ambiguously located machinery.

Other possible causes for ambiguity are locations on the underside or edge, of arches in walls that run obliquely, or almost orthogonal to the picture plane:

Piranesi 1: the two points at medium height left;

Piranesi 3: point at right, two-thirds from bottom.

Most of the remaining cases have to do with ambiguously located architectural details:

Piranesi 1: the leftmost point;

Piranesi 2: two balustrades at top right;

Piranesi 3: point at bottom left (the detail sticks out, but how much)?

Thus, virtually all of the ambiguous points do indeed pose problems, even in reflective thought (notice that the judgments during the sessions rarely took more than one or two seconds).

An intuitive manner to visualize the ambiguity is to compute fuzzy membership functions for the relation of any point to the fiducial point. We consider three sets, the 'hither' set, composed of points closer than the fiducial point, the 'here' set, composed of points at the same depth as the fiducial point, and the 'yonder' set, composed of points farther away than the fiducial point. Ideally, all memberships would be either zero ('not a member'), or one ('is a member'). In practice, a point may (simultaneously!) be a little closer, a little farther, and a little at the same depth (the memberships adding to unity) relative to the fiducial point. These functions yield an excellent impression of the incoherence (by way of example, see Fig. 9).

\subsection{Experiment 4: Single Observer Variability}

Observer AD completed six 'independent' sessions (weeks in-between) on the Piranesi stimuli (for example, see Fig. 10). The quartile interval of the figures of merit for the individual sessions is 0.874-0.901 (full range 0.840-0.913). (See Fig. 11, notice the strong overlap.) After finding the overall best linear depth order for each stimulus we find determinacy indices (with respect to the overall order). The quartile interval is $0.813-0.915$. The conclusion is that the sessions apparently form a rather consistent set, without obvious outliers.

We can also obtain fuzzy membership functions for the hither, here and yonder sets based upon the six sessions by observer AD. In Fig. 12 we show them for the same point that was illustrated in Fig. 9. It is apparent that the repeated results for a single observer tend to be more coherent than those for a group of diverse observers. 

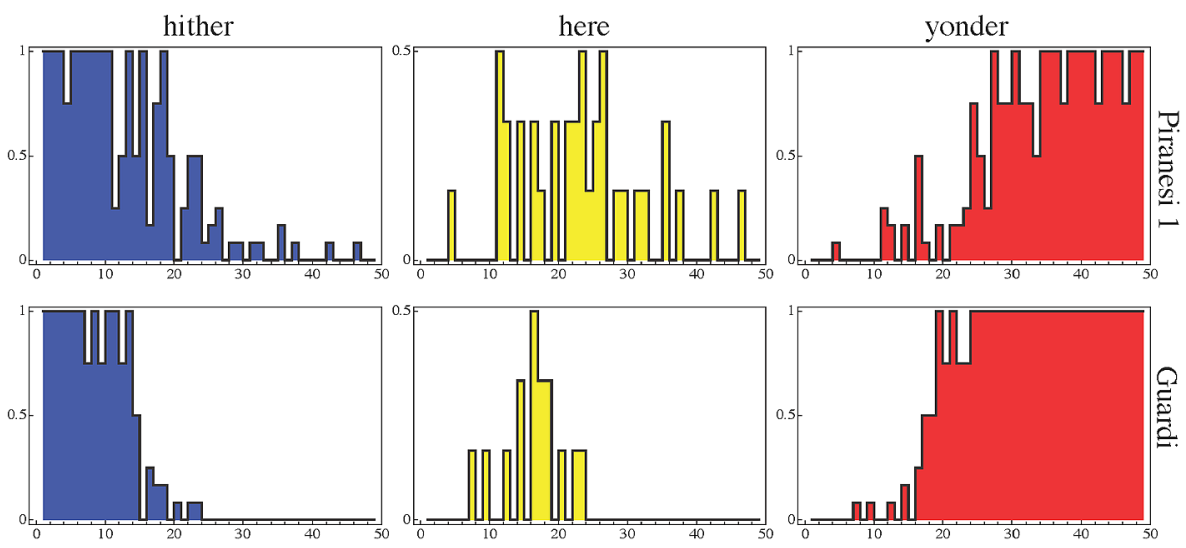

Figure 9. Fuzzy characteristic functions for 'hither', 'here' and 'yonder' for points in the Guardi and in Piranesi Stimulus 1, each based upon one session by six observers. In both cases we picked one of the ambiguous points. (On the horizontal axis the rank in the linear depth order, on the vertical axis the fuzzy membership.) The point on the Guardi is the rear of the boat in front of the scene. Most of the fuzziness is due to the other point at the front of the boat, the three points on the boat at right, and the points on the figures near the tent. The point in the Piranesi Stimulus 1 is the one on the tip of the beam that carries the central hanging rope. The fuzziness is widely spread over the middle ground.

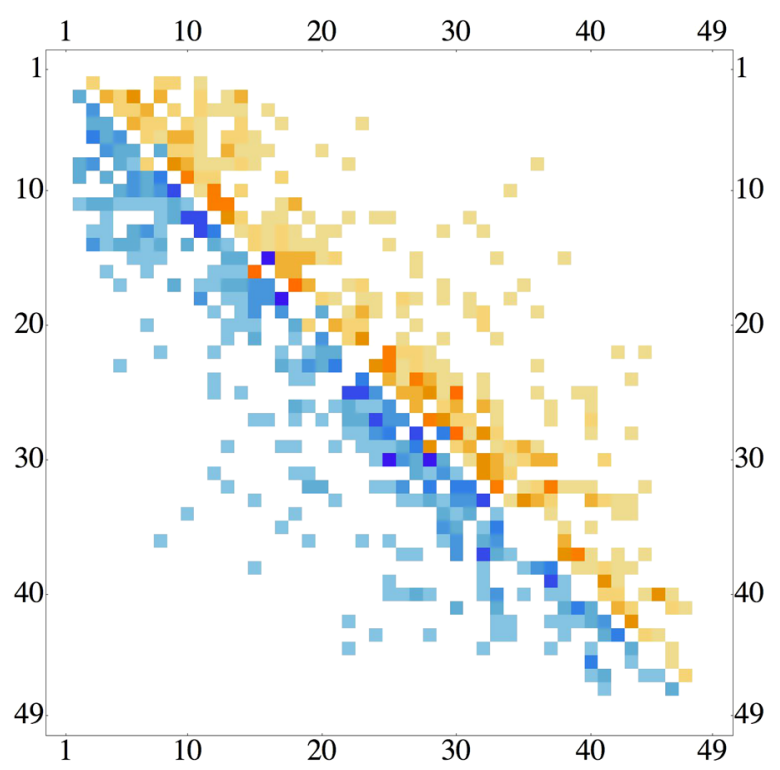

Figure 10. The mismatches for observer AD, Piranesi Stimulus 1, over six sessions. A 'mismatch' is a failure of the prediction based on the overall best linear order, with the individual judgments. 


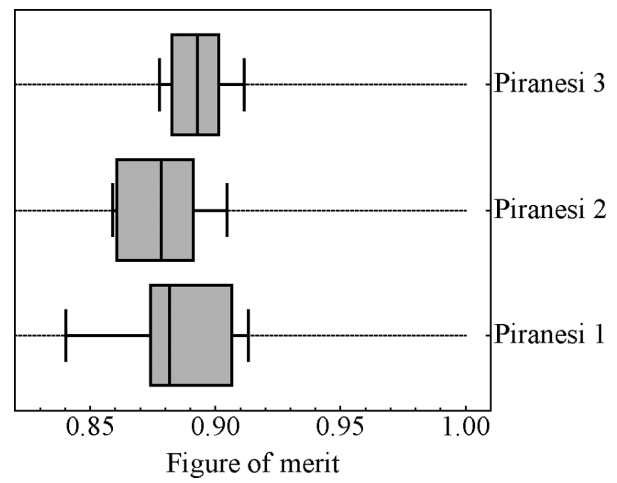

Figure 11. The distribution of the (single session) figures of merit for observer AD over six sessions for Piranesi Stimuli 1, 2 and 3. The whisker box plots show quartiles and extreme values.
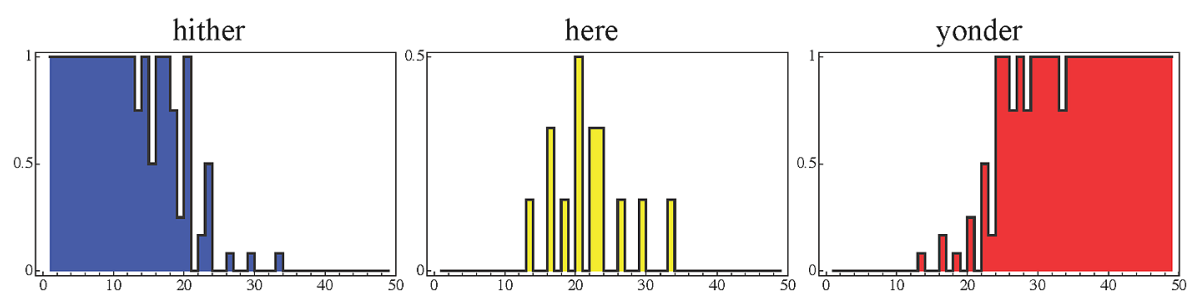

Figure 12. Fuzzy characteristic functions for 'hither', 'here' and 'yonder' for a point in the Piranesi Stimulus 1, based upon the six sessions by observer AD.

In Fig. 13 we show the hither, here and yonder sets for a single session of observer $\mathrm{AD}$ for the Guardi. In this case the memberships are (trivially!) not fuzzy, thus the result looks perfectly coherent. In a single session one needs 1176 judgments in order to be able to judge consistency, whereas in repeated sessions the fuzziness already appears for a single point, involving 48 comparisons.

The difference between the Guardi and the Piranesi stimuli (we regard Piranesi Stimulus 1 here) is also evident from the variation in judgments for a single observer over sessions. For six sessions we compute the consistency for the judgment of a single pair as fraction consistent answers (thus, both six times 'further' and six times 'closer' yield one, fifty-fifty 'further' and 'closer' yields zero, and so forth). By counting, we find the frequency for a given consistency. The values are displayed in Table 1 .

Thus, the judgments are much more variable in the case of the Piranesi than in the Guardi.

Another, different measure of consistency is to correlate the lists of judgments for two sessions. For the six sessions of AD for the Piranesi stimuli we find a range of values for the coefficient of determination of $0.571-0.734$ 


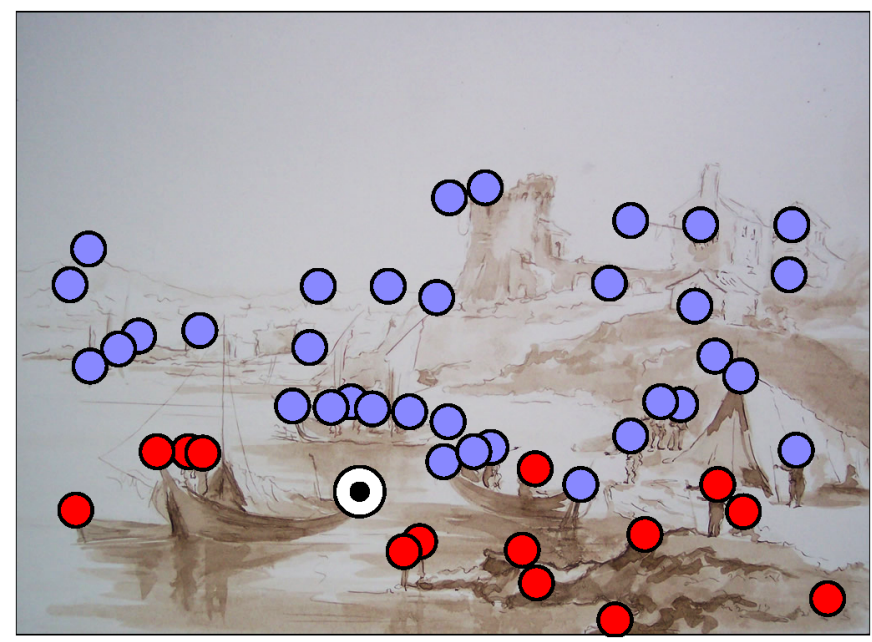

Figure 13. The points judged closer (in red), and farther away (in blue) than the fiducial point (larger, with black center dot) for one session of observer AD.

\section{Table 1.}

Relative frequencies of different levels of consistency between multiple sessions for one observer (see text for details)

\begin{tabular}{lcccc}
\hline Consistency & 1 & $\frac{2}{3}$ & $\frac{1}{3}$ & 0 \\
\hline Guardi & 0.878 & 0.084 & 0.025 & 0.013 \\
Piranesi 1 & 0.788 & 0.116 & 0.066 & 0.030 \\
Piranesi 2 & 0.766 & 0.120 & 0.080 & 0.034 \\
Piranesi 3 & 0.804 & 0.099 & 0.065 & 0.031 \\
\hline
\end{tabular}

(Piranesi 1), 0.574-0.668 (Piranesi 2), and 0.600-0.731 (Piranesi 3), quartiles 0.619, 0.678, 0.703 (Piranesi 1), 0.600, 0.626, 0.651 (Piranesi 2), and 0.640, $0.654,0.667$ (Piranesi 3). For the sessions of the six individual observers (only for the case of the Piranesi Stimulus 1) these values are much lower: the coefficient of determination ranges over $0.287-0.508$ (quartiles $0.355,0.384,0.430$ ). The highest value for the multi-observers case is lower than the lowest value for the six sessions of observer AD. Thus, observer AD is much more consistent over sessions (despite the long pauses between sessions) than the set of six observers between each other.

\section{Conclusion}

In this study we compared the coherence of pictorial space for a generic landscape rendering (a Capriccio, rather than a geographical location) by Francesco Guardi, evidently designed to be 'easy on the eye', and sheets 
from Giovanni Battista Piranesi's Carceri d'Inventione (second edition) that have become famous for their (intentional!) ambiguity, and are often denoted 'labyrinthine'.

We used a simple pairwise depth comparison task, considering all possible (orderless) pairs taken from forty-nine locations roughly uniformly distributed over the area of the prints. The points were located on obvious 'landmarks' and distributed over depth layers. Six observers participated in the experiments.

As might have been expected we find strong quantitative evidence for the notion that the Piranesi sheets are very ambiguous. Observers frequently disagree among each other, and (in repeated sessions) with themselves. Even results from single sessions are internally much less consistent for the Piranesi prints than for the Guardi.

Yet, one should not conclude that the Carceri prints appear as fully incoherent labyrinths. Indeed, this cannot be, for 'fully ambiguous' implies total chaos, which would essentially be without visual interest. The Carceri are sufficiently coherent to claim attention, yet sufficiently ambiguous to defeat attempts to arrive at a unique pictorial 'solution'. This, no doubt, accounts for (at least part of) their fascination. Thus, we find that the observers certainly can perform the task, and that one might even say that their results do show a remarkable degree of consistency given the ill repute of the Carceri sheets. It is not that the observers are fully lost, it is more that their pictorial spaces are much more 'fuzzy' than they are for easy targets like the Guardi Capriccio.

The tension between trivial chaos and trivial certainty no doubt (at least partially) accounts for the popularity of the Carceri. The Carceri manage to hit the range of highly non-trivial ambiguity. They keep the observer's microgenetic processes active, forever defying any permanent 'solution' (Pepperell, 2011; Van de Cruys and Wagemans, 2011a, b). This is a vital element of artistic appreciation, although rarely explicitly mentioned in discourses on aesthetics, and even more rarely targeted in formal research.

A final remark involves possible applications of our research. In Fig. 14 we plot the overall results of six observers. The large white disk with black center dot is a fiducial point; all other points are colored according to their depth relation from the fiducial point: red is closer, blue is farther away. Clearly the communis opinio is rather fuzzy. Apparently we cannot count on the notion that observers are aware of the same pictorial spaces when they look at the Piranesi sheet.

Notice that this latter finding has important implications in many potential applications. Even when observers happen to look at the same picture simultaneously, one may not (as is usually silently done) assume that they are aware of the same - or even similar - pictorial spaces. There is no doubt that this will influence the resulting discourse. 


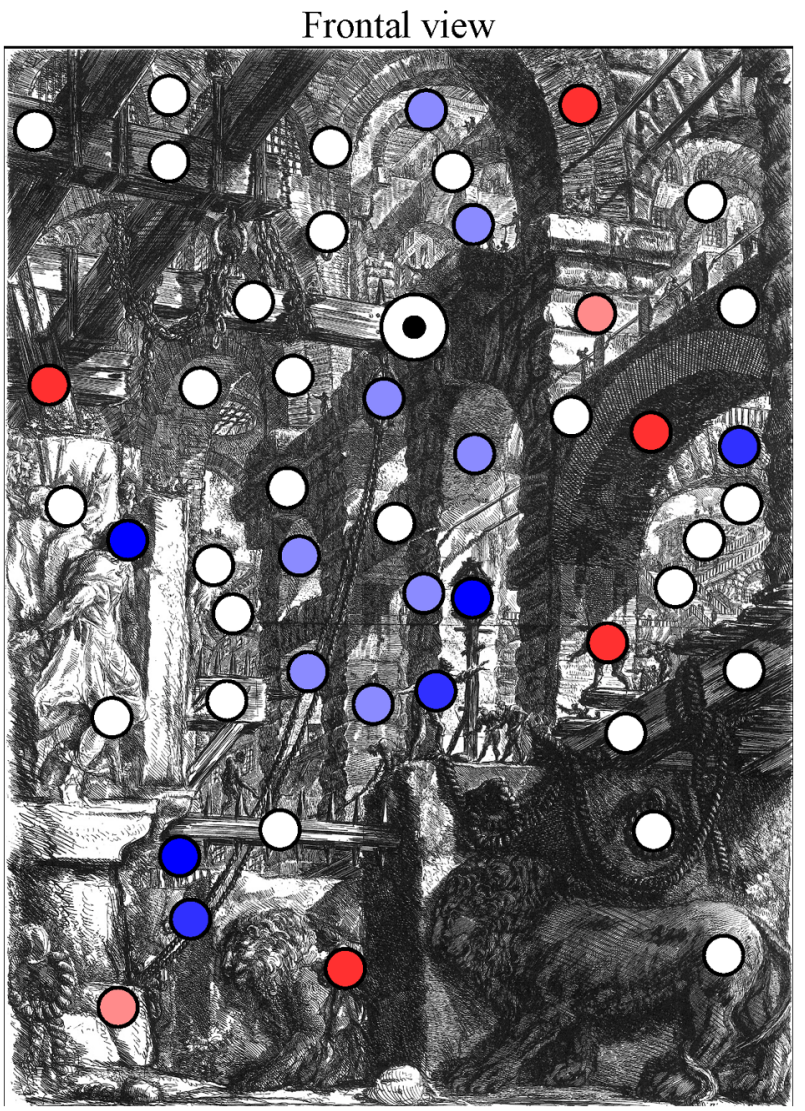

Figure 14. The points judged closer (in red), and farther away (in blue) than the fiducial point (larger, with black center dot) for the combined result of six observers. (We use hue to suggest fuzzy membership.) Notice how 'fuzzy' this result looks; a 'clean' result would look like Fig. 13.

\section{Acknowledgements}

This work was supported by the Methusalem program by the Flemish Government (METH/08/02), awarded to Johan Wagemans. We would like to acknowledge administrative support by Stephanie Poot, and technical support by Rudy Dekeerschieter.

\section{Notes}

1. On the Piranesi Carceri and their history see the Wikipedia site http://en.wikipedia.org/wiki/Giovanni_Battista_Piranesi. Good reproductions are available at http://www.let.leidenuniv.nl/Dutch/Renaissance/ Facsimiles/PiranesiCarceri1750/index.htm, and http://www.sanderusmaps. 


\section{com/en/our-catalogue/detail/166149/first-edition-of-piranesi's-carceri- d'invenzione/.}

2. The copy of the Guardi Capriccio can be found on http://www.atelierbonno.fr/peinture/galerie-art-grahique/guardi-paysage-imaginaire-2.jpg.

\section{References}

Ficacci, L. (2000). Giovanni Battista Piranesi: The Complete Etchings. Cologne and Rome.

Gombrich, E. H. (1961). Art and Illusion: A Study in the Psychology of Pictorial Representation. Princeton University Press, Princeton, NJ, USA.

Graham, C. H. (1965). Vision and Visual Perception. John Wiley and Sons, Oxford, U.K.

Hildebrand, A. (1893). Das Problem der Form in der bildenden Kunst. [The Problem of Form in Painting and Sculpture.] Transl. M. Meyer and R. M. Ogden (1945). G. E. Stechert, New York, USA.

Hofer, P. (1973). The Prisons (Le Carceri) - The Complete First and Second States. Dover Publications, New York, USA.

Koenderink, J. J. and van Doorn, A. J. (1995). Relief: pictorial and otherwise, Image Vision Comput. 13, 321-334.

Koenderink, J. J. and van Doorn, A. J. (2003). Pictorial space, in: Looking into Pictures: An Interdisciplinary Approach to Pictorial Space, H. Hecht, R. Schwartz and M. Atherton, Chapter 12, pp. 239-300. MIT Press, Cambridge MA, USA.

Koenderink, J. J., van Doorn, A. J. and Wagemans, J. (2011). Depth. i-Perception (special issue on Art and Perception) 2, 541-564. doi:10.1068/10438aap.

Koenderink, J. J., van Doorn, A. J. and Wagemans, J. (2012). Picasso in the mind's eye of the beholder: Three-dimensional filling-in of ambiguous line drawings, Cognition 125, 394412.

Pepperell, R. (2011). Connecting art and the brain: an artist's perspective on visual indeterminacy, Frontiers in Human Neuroscience 5, doi:10.3389/fnhum.2011.00084.

Pirenne, M. H. (1970). Optics, Painting and Photography. Cambridge University Press, Cambridge, U.K.

Van de Cruys, S. and Wagemans, J. (2011a). Gestalts as predictions: Some reflections and an application to art, Gestalt Theory 33, 325-344.

Van de Cruys, S. and Wagemans, J. (2011b). Putting reward in art: a tentative prediction error account of visual art, i-Perception (special issue on Art and Perception) 2, 1035-1062. doi:10.1068/i0466aap.

van Doorn, A. J., Koenderink, J. J. and Wagemans, J. (2011). Rank order scaling of pictorial depth, i-Perception (special issue on Art and Perception) 2, 724-744. doi:10.1068/i0432aap.

von Helmholtz, H. (1856). Handbuch der physiologischen Optik, 1st edn. Leopold Voss, Leipzig, Germany.

Wagemans, J., van Doorn, A. J. and Koenderink, J. J. (2011a). Measuring 3D point configurations in pictorial space, $i$-Perception 2, 77-111. doi:10.1068/i0420.

Wagemans, J., van Doorn, A. J. and Koenderink, J. J. (2011b). Pictorial depth probed through relative sizes, $i$-Perception 2, 992-1013. doi:10.1068/i0474. 